\title{
Strategic Challenges in Neurotherapeutic Pharmaceutical Development
}

\author{
Christopher C. Gallen \\ Clinical Research and Development, Wyeth Pharmaceuticals, Collegeville, Pennsylvania 19426
}

\begin{abstract}
Summary: Developing new therapeutics presents formidable economic, scientific, and medical challenges that are exacerbated by special factors in neurotherapeutics development, e.g., the complexity of the CNS with its attendant need to sometimes affect multiple pathways, the lack of clarity of disease etiology, inadequacy of available animal models, and difficulties in defining disease populations and quantifying treatment response. This paper reviews the economic challenges faced by therapeutics developers in general and neurotherapeutics developers in particular. It discusses key scientific challenges, particularly those pertinent to neurotherapeutics development, as a background to proposing a different industrial strategy to drive future therapeutics development. This Biodesign strategy potentially surpasses previous paradigms by incorporating ele-
\end{abstract}

ments such as the use of disease modeling to select better targets and potential therapies, information science-based approaches to enhance small molecule chemistry, exploitation of the potential for biological technologies to rapidly generate mechanistic probes, and development of improved approaches for using animal models and studying human molecules mechanistically and biologically. Synergistic use of these elements can change the overall business model of companies engaged in neurotherapeutics development. The Biodesign paradigm has the potential to both markedly enhance the development of new therapies and to address some of the economic challenges facing healthcare systems and therapeutics developers alike. Key Words: Neurotherapeutics, pharma, disease models, proteins, small molecules.

\section{INTRODUCTION}

The pharmaceutical industry must generate novel, effective, and safe therapeutics that address unmet medical needs at a cost that is palatable to consumers globally, in a time frame that allows effective recovery of the investments in the systems and processes necessary to generate new products, and in a manner compliant with international regulations.

In the long run, return on investments in therapeutics development must pay for the full economic costs of development or the process will fail economically and cease. The seriousness of this challenge can be inferred from the steady attrition of pharmaceutical companies large and small, old and new. The economic component grows steadily each year because of increasing pricing pressures, costs, generic competition, and regulatory hurdles, along with decreasing productivity.

The scientific challenges for therapeutics developers are great in every area but particularly so in neurothera-

Address correspondence and reprint requests to Christopher C. Gallen, Wyeth Research, 500 Arcola Road, Collegeville, PA 19426. E-mail: gallenc@wyeth.com. peutics. On one level, new scientific discoveries and tools create the possibility for a golden age of therapeutics development, in which many long-term scourges of humankind may be addressed in a fundamental and powerful manner. Yet while we move from symptomatic treatments to mechanistically targeted cures, we struggle to base development on fields of knowledge that are rapidly changing and far from complete and in which our understanding of the interactions between factors is fragmentary at best. Neurotherapeutics developers face unusually high failure rates and huge attendant expenses that must be covered by the small number of therapeutics that succeed.

The combination of pricing constraints and trends of increasing regulatory hurdles, falling productivity, lengthening cycle times, and falling success rates is not sustainable in the long term. Many developers have focused on strategies to improve scientific and operational performance, such as improved collaboration with academicians and regulatory agencies, better use of technologies to explore study data and design trials, greater operational effectiveness in selecting and training quality sites, monitoring and harvesting data, producing analy- 
ses, and amortization of costs over a wider base by global registration. Although important, these approaches are not enough. Issues of discovery and clinical development strategy must also be addressed. These issues are particularly pertinent to neurotherapeutics development and even more so to the development of therapeutics for neurodegenerative disorders.

A new and better paradigm must be developed for pharma to succeed economically and medically in the long term. While incremental improvement of current approaches can be useful and necessary, a more powerful and deeply penetrating change is needed to meet the challenges the therapeutics development community faces. In the last century, the pharmaceutical industry progressed from a strategy of observation and serendipity to the current strategy of rational design, which argues that mechanistic understanding of disease leads to mechanistically targeted molecules, which works if the theory of disease etiology is correct. The conceptual weakness of this strategy is that the map is not the territory. The systems biology underlying disease is complex, the relevant mechanisms are often multiple, interactive, and variable across species, the individual variability in an outbred human population is great, and the statistical noise generated by imperfect clinical assessment instruments together are a huge barrier to overcome. Narrowly targeted molecular interventions based on simplistic mental maps of the disorder sometimes work but often run afoul of these complicating factors.

The next strategic approach, Biodesign, potentially surpasses the performance of the rational design strategy by embracing complexity by using the capacity of the immune system to rapidly generate the myriad potential probes needed to assess the therapeutic potential of different interventions in conjunction with in vivo assessment technologies targeted to the human disease model and integrated with computerized disease models. Elements of this proposed paradigm include the use of disease modeling to select better targets and potential therapies, along with information-science-based approaches to enhance small molecule chemistry, exploitation of the potential for biological technologies to rapidly generate mechanistic probes, and development of different strategies for using animal models and enhanced strategies and abilities to study human molecules mechanistically and biologically.

The synergistic use of these approaches will change the overall business model of companies engaged in the development of neurotherapeutics as well as other therapeutic areas. The next-generation pharmaceutical company will be defined by intense, deep, and advanced focus on specific target diseases, working with defined populations and specialists to target disease mechanisms with ever-broadening power. The model will combine expertise in protein therapeutics and small molecules and use them interactively in devising commercially viable therapeutics. This development will both depend on and generate a new set of relationships and interdependencies between academicians, regulators, and industry. In so doing, the Biodesign paradigm may revolutionize medicine and solve the economic challenges facing healthcare systems and therapeutics developers alike.

\section{THE TERRAIN AND THE PATH FORWARD}

In determining the strategy best suited to creating the next and better generation of medical therapies, it is critical begin with a map-a conceptual picture of the terrain, the challenges and obstacles-so that the most effective path forward can be plotted and followed. This essay will focus on describing key elements that must be overcome by next-generation industry strategy because pharmaceutical development sits at the intersection of two different concerns. First, there must be an unmet medical need of sufficient magnitude as to potentially justify the investments required to generate a novel therapeutic. Second, the basic science or a key observation must exist to support the hypothesis that some mechanism or molecular target or target process relevant to a disease exists and is sufficiently defined as to permit discovery approaches to operate with some confidence of a potentially successful outcome. When there is an existing and potential market and a clear scientific strategy to produce clinically meaningful benefit, industrial processes come into play.

Neurotherapeutics development exists within the larger context of general pharmaceutical development but has unique scientific and economic challenges and opportunities that make it one of the most demanding and high-risk areas for pharma. (Note that the term pharma is meant in a broad and inclusive sense primarily to define both the pharmaceutical and biotechnology industries, although many issues are equally relevant to device therapeutics.) The economic opportunities may be great, but success rates are appallingly low, costs of development are high, and timelines for return are unusually long.

\section{THE OPPORTUNITY FOR NEUROTHERAPEUTIC PHARMACEUTICAL DEVELOPMENT}

The pharmaceutical industry was born in the 19th century through interactions among analytical chemistry isolating active compounds from natural substances, organic chemistry becoming increasingly sophisticated in modifying those compounds and producing novel therapeutics, and the progress of experimental pharmacology. ${ }^{1}$ Historically the industry has evolved with help from serendipity, hard work from many and singular genius 
from a few. The early successes of the chemotherapeutics era centered on antibiotics. In the mid-20th century, technological and conceptual advances in biochemistry triggered an explosion of therapeutics targeted towards protein receptor and enzyme-target agents. More recently, advances in biotechnology have triggered a creative burst of protein therapeutics.

Technologies for developing therapeutics have improved dramatically. X-ray crystallography and other structural approaches help define target shapes with angstrom accuracy, allowing for more rational design and, at its best, virtual chemical design. Automated combinatorial chemistry vastly speeds the ability to generate new small molecules to test against those targets. Various types of assay chips make it possible to perform tens of thousands of screenings in an hour. The combination of a vastly increased array of targets through genomics, the ability to generate large numbers of potential ligands through combinatorial chemistry, and the ability to conduct automated high-throughput screenings through in vitro or cell-based assays potentially creates a situation in which the magic of large numbers may potentially be more productive of therapies than the older system, emphasizing scientific reasoning and critical discourse between chemists and biologists. ${ }^{1}$ The recent description of the human genome and the rapid developments in understanding how it provides cells with instructions to produce as many as 300,000 different proteins ${ }^{2}$ holds the promise of allowing a fundamental understanding of the molecular logic of the life process, thereby creating myriad openings for highly targeted and possibly personalized therapeutics. The development of brain imaging technologies such as positron emission tomography (PET) and functional magnetic resonance imaging opens the door to more direct and quantitative understanding of drug effects in humans. Looking at what has been accomplished and at the ongoing stream of progressive improvements across the board, the historical vector points to a future with remarkable possibilities for addressing major human health problems in all therapeutic areas, including neurotherapeutics.

\section{ECONOMIC CHALLENGES FACING THERAPEUTICS DEVELOPERS}

\section{Overall economic success is a requirement}

Human behavior is typically driven by a complex combination of competing and complementary motives. Such motives, which may include interest in science, personal experiences or concerns, economic drives for reward, and humanitarian motives, drive resource allocation and aggressiveness in pursuing goals for any given therapeutics developer. The magnitude of investment required for success is so huge that it is understood that economic motives must and-from a societal resource allocation perspective-should play a strong role. The very fact of having sufficient resources to pursue large-scale development implies economically prudent decisions in the past to generate those resources. Rational economic investment presupposes some hope of reward that must be balanced against risks. If there is huge risk in pharmaceutical development, the real impact of successful therapeutics on human lives and national economies is also huge and therefore pharmaceutical development is potentially filled with rewards that drive the economics of investment.

Unfortunately, in therapeutics development, it is impossible to predict with any certainty whether a given effort will succeed or fail, therefore costs will be incurred for both successful and failing programs. For the overall pharmaceutical development enterprise to continue in the long term, the income that ultimately results from the successful development efforts considered in aggregate must compensate for the costs of and capital for both the successful and unsuccessful efforts. The same is true for individual companies. If the revenues produced by successful products do not compensate for the fully loaded costs of development, the costs of capital, and some profit incentives, the failing enterprise will eventually become extinct. The difficulty of meeting this basic condition can be inferred from the large number of pharmaceutical companies that have failed and disappeared or been acquired. Companies may merge for reasons of synergy and/or cost reduction, but the reality is that many mergers are driven by the need of endangered or nonviable entities to find a stronger partner to secure whatever residual value their assets contain, values that would be completely lost if the company went under. Conversely, the more profitable the successful efforts are, the more resources available to explore a wider range of potential projects, including inherently riskier novel approaches. Where successful efforts are less profitable, fewer resources are available to explore novel approaches and attention tends to focus on activities that are perceived as lower risk, such as the development of therapeutics based on more proven mechanisms (often derided as "me too," but conferring some valuable marketplace choices), lifecycle management of existing products, and acquisition of products for which better marketing or combination with the existing business holds the promise of commercial reward.

\section{The economic reward}

The human and economic burden from CNS disease is great and drives the need for significant investments in therapeutics development. Size estimates of the CNS market depend in part on the entities considered as neurotherapeutics, e.g., neurologic, psychiatric, pain, anesthetics, or drug addiction and abuse agents. In general, 
sales of CNS therapeutics comprise approximately $15 \%$ of total pharmaceutical sales, approximately $\$ 30$ billion worldwide. About two-thirds of these sales are for psychiatric treatments; historically, more mechanisms leading to effective products have been discovered relevant to psychiatric illness than to neurologic illnesses. Effective CNS pharmaceuticals have the potential to provide a huge benefit to patients and economies. For example, the estimated annual economic costs of anxiety disorders, depression, and schizophrenia are $\$ 47$ billion, $\$ 44$ billion, and $\$ 33$ billion per year, respectively. ${ }^{3}$

These numbers reflect the current market but may vastly understate the potential market. First, many important CNS disorders have no curative treatment at all; thus if a treatment is developed, an entirely new market comes into play. Second, other disorders have only ameliorative therapies that either have limited efficacy or are associated with significant side-effects that strongly restrict their use. More effective or better-tolerated safer therapies have the potential to dramatically increase utilization and therefore market size. Third, markets currently served by workable but suboptimal generic therapies have the potential to grow dramatically with the introduction of more effective or safer proprietary therapeutics. In assessing the economic potential of any prospective therapeutic, the unmet medical need, the number of potential patients who could be served by a safe and effective therapy, the seriousness of the illness, and the disability entailed must be considered (and all affect the potential benefit and hence the price of a therapy). An important concern arises from these assessments. While very welldeveloped markets such as the market for antidepressants, anti-epilepsy agents, or anxiolytics are well-understood and valued, the market worth for diseases in which current therapeutics have had less impact, such as stroke or dystonia, is less clearly defined. In this situation there is the potential for underestimation of market size and consequent underinvestment.

\section{The economic risk}

A few general factors control whether development of a particular therapeutic is economically feasible. The key economic determinants of reward are the size of the market, the real competitive advantages conferred by the potential new therapeutic, its anticipated patent life ("marketing life") and potential price, and the number of competitors already occupying the market. The key economic determinants of risk are success rate, development time, and development cost.

In general therapeutics development, for every 5,000 to 10,000 compounds screened, typically about 250 will enter preclinical testing; of those, five will enter clinical testing and one will win approval by the U.S. Food and Drug Administration (FDA). ${ }^{4}$ Overall, only about $11 \%$ of new active substances entering clinical development are predicted to reach the market. ${ }^{5}$ The success rates for neurotherapeutics, however, are far lower than average. The relative difficulty of neurotherapeutics development is illustrated by a comparison of the chance of compounds initiated into human testing to progress to eventual marketing across therapeutic areas: anti-infectives, $33 \%$; cardiovascular, $6 \%$; anti-cancer, $6 \%$; and nervous system, $1 \% .^{5}$ Of equal concern is the fact that neurotherapeutic compounds fail late in development (during phase 3 pivotal testing) far more often than other compound categories. The chance that compounds initiated into pivotal trials will subsequently progress to eventual marketing across therapeutic areas is higher, but nervous system compounds are still dramatically riskier: antiinfectives, 75\%; cardiovascular, $43 \%$; anti-cancer; $32 \%$; and nervous system, $14 \% .^{5}$ As a rule, pivotal programs cost about three times as much as the combined cost of phase 1 and 2 trials. Low success rates late in development hugely accelerate the financial risk of neurotherapeutics development. Of pharmaceuticals that do win approval, only one in three will produce revenues that match or exceed development costs. ${ }^{6}$ The revenue stream and profits produced by one pharmaceutical will basically have to pay for the tens of thousands of antecedent compounds produced, screened, and rejected along the way.

The length of development time is a key parameter governing economic risk for the developer. Because the ability to recoup the massive investments required to develop a pharmaceutical usually drops dramatically once it goes off patent, the costs of development must be recouped while the product is on patent. Longer development times directly reduce the number of profitable years remaining for any therapeutic. In the United States, it takes 10 to 15 years to move a new therapeutic from discovery through regulatory approval: around 4 to 6 years for the discovery/preclinical phase, around 4 to 6 years for the clinical phase, and 1 to 2 years for the initial regulatory approvals. In a patent life of 20 years, that leaves only 5 to 10 years to recoup the fully-loaded costs of development and capital. Median development times vary more on the basis of indication rather than target organ, i.e., analgesics development is comparatively rapid but progressively longer times are required for neuroleptics, Alzheimer's disease therapies, antidepressants, and stroke treatments. The two biggest factors leading to prolonged development times are the time required to enroll enough patients for studies to have statistical significance and the treatment period needed to detect an effect. Both factors are particularly acute in neurotherapeutics development; most therapeutics for neurodegenerative diseases tend to require relatively prolonged periods of treatment and observation to detect an effect. Additionally, the real cost of a development program includes the costs of capital, that is, the income that 
could have been derived had the funds been invested in a different, lower-risk investment. Long development times raise capital costs. Analyses by the Tufts Center for the Study of Drug Development have indicated that reducing the total development time by half will reduce total costs by $29 \%$. $^{7}$

Development costs are a combination of fixed and variable costs. Extensive and expensive discovery resources are needed to explore mechanisms, probe interventions molecularly, develop therapeutic molecules and delivery mechanisms, and assess toxicology and other parameters in detail. Developmental testing of potential therapies in humans, as controlled by international regulations, principles of good clinical practices, and other constraints, involves extensive efforts to conceive development plans and protocols, conduct detailed testing in thousands of patients, capture and analyze all data in robust and reliable systems, and write extensive reports and submissions. Cost estimates for pharmaceutical development vary widely but were estimated by the Tufts Center to be approximately $\$ 802$ million in $2000 .^{8}$ This estimate included an average out-of-pocket cost per new drug of $\$ 403$ million plus costs of capital; it was further noted that the capitalized post-approval development costs raise the overall pre- and post-approval cost to $\$ 877$ million. ${ }^{9}$ This number includes out-of-pocket preclinical and clinical expenses and costs of capital for preclinical and clinical expenditures for the expenses of both project failures and successes. These costs have consistently been driven upward by the progressive increase in the number of clinical trials: from 30 in the period from 1977 to 1980 , to 68 in the period from 1994 to 1995 . Similarly, the number of patients per New Drug Application (NDA) has increased from 1,576 in the period from 1977 to 1980 to 4,237 in the period from 1994 to $1995 .{ }^{10}$ The $\$ 802$ million figure is likely conservative. As a cross-check, one could examine the research and development (R\&D) budgets of most major pharmaceutical companies, divide by the portion of the $\$ 403$ million related to direct expenses rather than costs of capitalization, and get a number of predicted compounds far higher than the average yearly number of new chemical entities registered by that company. Even if the $\$ 802$ million was considered as fully-loaded costs (including costs of capital) and divided into the R\&D budgets of the larger companies, the resulting number would be higher than the average number of new chemical entities registered by those companies per year. In 2000, for example, 11 major pharmaceutical companies had R\&D budgets greater than $\$ 2$ billion per year, yet based on current pipelines and success-rate estimates, predictions suggest launch rates averaging 1.3 new active substances per year over the past 6 years. ${ }^{11}$ As already noted, these numbers are of all the more concern when coupled with the realization that the $R \& D$ costs are such that only three in 10 marketed drugs produce revenues that exceed or match their development costs. ${ }^{12}$

\section{Industrial investment and productivity}

Pharmaceutical R\&D investments are high and growing geometrically. In 2002, members of the Pharmaceutical Research and Manufacturers of America spent approximately $\$ 32$ billion on pharmaceutical R\&D, which represents about a 15 -fold rise over the past 20 years and exceeds the National Institutes of Health (NIH) budget of $\$ 24$ billion. NIH funding is critically important for the general advance of health sciences and should not be underestimated, but a 2001 report by the NIH indicated that when specific links to pharmaceutical developments were assessed for 47 drugs with US sales of $\$ 500$ million or more per year, only four drugs had been developed in part with NIH-funded technologies. ${ }^{13}$ Domestic U.S. pharmaceutical $R \& D$ expenditures exceed those of any other major industrial sector, even high investment sectors such as computer software and services and the electrical, electronics, and aerospace industries.

Company-financed research in products affecting the CNS and sense organs was estimated at $\$ 7.3$ billion in 2001 , significantly exceeding the $\$ 3.9$ billion expenditure for agents acting on the cardiovascular system and roughly equal to the combined $\$ 7.4$ billion expenditure on products affecting neoplasms, the endocrine system, and metabolic diseases. ${ }^{14}$ More than $80 \%$ of larger pharmaceutical companies developing agents to treat CNS disorders. They focus primarily on larger, more welldefined indications such as depression, schizophrenia, and multiple sclerosis, or on underserved indications in which medical need is high, such as dementias, brain tumors, or substance use disorders.

Geometric increases in R\&D expenditures notwithstanding, the overall productivity of pharmaceutical research in producing new chemical entities has not increased in proportion to the investment. Over the past 20 years, pharmaceutical research has increased about $1,500 \%$ but the number of approvals of new therapeutic agents has been relatively small, rising from approximately 20 per year in the 1980 s to approximately 30 per year in the 1990s and currently. ${ }^{15}$ This failure of productivity has been partially ameliorated by the wider markets opened by globalization and regulatory developments that facilitate more global registrations of treatments. But the pressures of productivity challenges are exacerbated by both the competition from "fast followers" and the challenge to generate new therapeutics fast enough to replace those that go off-patent. The ability of new technologies to rapidly close discovery gaps once a promising new target for development is proven greatly facilitates the capacity of fast-follower companies to exploit the discoveries of innovator companies. This process is reflected in the progressively reduced 
period of marketing exclusivity and market share enjoyed by an innovator company before competitors match the initial breakthrough process. The huge economic importance of patent expiration is driven by the fact that generic production has taken an increasing share of the U.S. prescription pharmaceutical market. ${ }^{16}$ Once the patents constraining the generic use of a compound expire, the compounds are typically produced by a manufacturer with small costs to recover ( $\$ 1$ million to $\$ 2$ million for bioequivalence studies) and no requirement to meet the significant costs required for the development of next-generation pharmaceuticals. In a sense, the compound passes into the patrimony of humankind, where it is sold at markedly reduced prices that do not cover the costs of further research and development. Patent expiration is a huge challenge to innovator companies, in some cases engendering crisis and the risk of economic failure.

Many major pharmaceutical companies have recognized the need to double or triple their discovery output to maintain current profitability and growth in the face of generic competition. ${ }^{17}$ High throughput screening has been portrayed as a major and massive source of new compounds, ${ }^{1819}$ yet analyses suggest that actual utilization is at only $2 \%$ to $7 \%$ of installed capacity ${ }^{20}$ and is not likely the rate-limiting step. Later issues such as biometabolism and compound toxicology are more important limitations to discovery output and are being managed by industrializing the screening process.

Price increases are unlikely to play a significant role in compensating for the productivity challenge to the pharmaceutical industry. Because most of the expenses associated with pharmaceuticals derive from $R \& D$ and marketing costs rather than the specific cost of goods production, prices tend to be driven by the relative benefit conferred, not the cost of unit production (although in general the cost of goods is much higher for protein therapeutics than for small molecule therapeutics and can constitute a significant portion of the total price). Price pressures are high. Healthcare expenditures are a large budget item worldwide. In 1997, healthcare costs as a percentage of gross domestic product were higher in the United States than in other major industrialized nations. Pharmaceutical costs are a small percentage of overall healthcare costs, about $8 \%$ in the United States. On average, pharmaceutical costs are similar to the average telephone bill, but are of concern because they disproportionately affect vulnerable segments of the population such as the elderly. In most of the world, pricing is tightly controlled by governments at levels that are, in aggregate, not compatible with sustaining current worldwide pharmaceutical $R \& D$ expenditures. These prices tend to cause pharmaceutical research to shift outside the borders of the countries with lower pricing ${ }^{21}$ and to decrease availability of therapeutics to patients by slow- ing introduction of new therapies. ${ }^{22}$ It can be argued that pharmaceuticals may actually decrease overall healthcare costs by reducing larger expenditure items such as hospitalization. But the key strategic implication of this pressure for developers of neurotherapeutics and other drugs is the fact that increased costs of research and development are unlikely to be covered by price increases, and therefore the need to dramatically improve productivity is inescapable.

\section{SCIENTIFIC CHALLENGES FACING THERAPEUTICS DEVELOPERS}

\section{Genomics}

The mapping of the human genome offers a startling opportunity for the development of new generations of therapies in the long term. All of the understood therapies currently in use derive from a relatively small set of targets, about $500 .^{23,24}$ With the mapping of approximately 25,000 to 50,000 new targets and the recognition that there may be as many as 300,000 (or more) proteins of interest, the number of targets that potentially can be explored has expanded dramatically. The promise is for fundamentally targeted treatments, likely coupled with improved diagnostics to exactly determine the target defect in a given individual. This is very exciting, but the promise may be long in coming when one considers some of the challenges. Relatively little is known about most of these genes. This may be helped by the NIH Protein Structure Initiative as it identifies 10,000 protein structures over the coming decade. Similarly, the vast computational power of new supercomputers may speed progress in understanding the structure-function properties of proteins and thereby in facilitating the targeting of drugs to proteins.

There is an important caveat about even "simple" genetic abnormalities as mechanistic targets. Even a disease such as cystic fibrosis that is related to a single gene product has more than 1,000 known mutations, most of them private (related to a single family) and a few more common mutations that cause the disease in a larger number of individuals ${ }^{25}$; this is unsurprising. A given molecule may be dysfunctional because it is never made or has a different Michaelis constant (Km) well above the natural concentration of its molecular substrate, or has a maximum velocity $(V \max )$ well below normal, or has a normal $\mathrm{Km}$ and Vmax but is unusually subject to proteolysis and has a short half-life, or aggregates with other proteins differently, or is subject to some other disruptive factor. The key is to realize that each of these very different problems may not be solved by the same intervention. One dysfunctional molecule may be best remediated by an agent that stabilizes it, while another may need an allosteric modification to change its affinity for substrate. Similarly, in thalassemias patients with 
seemingly identical genotypes may differ greatly in severity. ${ }^{26}$ The protein deficiencies responsible for the hemoglobinopathies have been well-characterized for some years, yet the curative therapies are still some time away.

Genomics opens up the possibility of more effective therapeutics but for many conditions the needed interventions may in fact require significant individualization. The development of compounds targeting individualized therapies is likely to be highly un-economic, particularly if each such compound is required to meet current regulatory standards of proof, including the requirement for study in large numbers of patients. ${ }^{28}$ This challenge is even greater when it is noted that most of the largemarket nervous system diseases are thought to be polygenic. ${ }^{28}$ Additionally, the lack of complete concordance between twins in studies of important nervous system diseases such as schizophrenia, depression, and multiple sclerosis highlights the importance of environmental factors. In the long run, genomics is of great importance in providing targets and a basis for a better understanding of which targets matter, but the complexity of the challenge is sufficient that the long run may too long for some developers to last.

\section{Specificity}

The special challenges of neurotherapeutics development begin with the unusually complicated, anatomically specific, and temporally intense nature of the functioning of the target organ: the brain. Although all organ functions, from heart and liver to kidney and lung, have significant inherent complexity, the complexity of the brain is particularly immense. In the midst of normal functioning, neurons with patterns of connectivity related to development but also affected by antecedent experience interact via intimate connections and communicate signals based on the fine timing and code of the firing. The excitation of any one neuron is typically the result of convergent stimulation from other neurons and in turn can affect multiple other neurons. Signals flow simultaneously to excitatory and inhibitory recipients to then be transmitted back to the source neuron as a regulatory signal or to affect the firing of other neurons in parallel or other pathways. Moreover, the system self-regulates over time to compensate for changes in input. Because the bursts of stimulation leading to neuronal firing are brief, while pharmaceutical levels typically vary slowly over hours, there is no exogenous chemical delivery system that could directly excite and inhibit specific targeted neurons in the timing pattern to allow direct mimicking of neuronal function. There is no exogenous chemical targeting system at this time that can directly stimulate or blockade only a highly specific cluster of neurons while avoiding effects on other neurons of the same class where the same signal might have a different and unwanted effect. Instead, there are two basic options: to essentially raise or lower the general signal strength in a given system by the application of agonists or antagonists and allow the fine pattern of signal transmission that was otherwise present to continue in an amplified or subdued manner, or to enhance or retard the release, removal, or degradation of an endogenous transmitter to retain its pattern of release while amplifying or diminishing its signal. In principle, combinations of invasive mechanical and chemical delivery systems might accomplish greater anatomical specificity but would still act at a gross level. In some cases, the nature of the system or the specificity of the receptors is such that a relatively specific influence over a more general system is possible. For the most part, however, pharmaceuticals circulating to the brain have the potential to produce widespread effects across the brain and the body rather than only focusing the desired action on a particular nucleus or nuclei.

As a further complication, the functioning of many neuronal systems and the pathology in many neurologic diseases involve complex systems influencing more than one neurotransmitter. The implication is that highly specific effector molecules may influence one set of neurons that are part of a disease process but in their very specificity fail to affect other systems that are also a part of the same disease process. The normal course of discovery assessment of molecules generally proceeds on the principle of creating the most selective molecule-agonist or antagonist-possible. Modification of a given brain system by a specific effector molecule produces potential adverse events by stimulating the same target in other brain regions. For example, the blockade of dopamine neurons by dopamine antagonists that produces beneficial effects on schizophrenia by affecting the mesolimbic dopaminergic systems also produces adverse effects on prolactin levels by blocking tuberoinfundibular dopaminergic neurons. But because more than one system may be relevant to a given disease process, the very specificity designed into the molecule by discovery groups also precludes the possibility of affecting those other neuronal systems relevant to the disease. In other words, the effort to create a molecularly clean and specific "bullet" is likely to result in decreased efficacy in a situation where more than one neurotransmitter system is involved in the pathology. It is perhaps not a coincidence that clozapine, the prototype atypical neuroleptic believed by many to be the most effective agent for treatment-resistant schizophrenia has potent anticholinergic, antiadrenergic, antihistaminic, and antiserotonergic properties.

In principle, one could develop a series of specific effector molecules to mix and match in polypharmacy to optimally treat a particular disease or to optimize treatment for an individual; such a system could allow optimization of the exact amount of blockage or facilitation of each relevant system in a given individual to optimize 
response and reduce adverse events. The reality, however, is that to be registered for therapeutic use, each molecule would have to be approved independently as a therapeutic. Because the more specific molecules are often likely to be at a therapeutic disadvantage to less specific molecules already on the market that serendipitously affect a better combination of targets, these approvals would be very difficult to obtain. Hence the option of producing patient-specific or disease-specific cocktails that combine the right set of agonists and antagonists seems unlikely. Indeed, by the time all the approvals had been obtained on all the relevant cocktail components, the patent life would likely have expired on some. For small molecules at this time the field instead seems largely stuck on developing molecules that affect multiple systems in some relative ratio fixed in the design of the molecule, in the manner of the design development of the various atypical neuroleptics.

Another aspect of specificity relates to the ability of discovery groups to produce molecules that effectively interact with new genomic targets. In current discovery functioning, as a target is identified the company library of compounds-hundreds of thousands to millions of compounds-is screened against the target. Compounds that assay well are studied as possible lead structures and the most promising are modified and tested, then further modified iteratively until optimum compounds are developed. Such compound libraries historically have been generated by prior programs and as such tend to be heavily weighted toward compounds hitting G-proteincoupled receptors, serine proteases, and similar targets and may not always have the structural diversity and range of structural complexities that are optimal to meet entirely new classes of targets uncovered by new genomic approaches. Improving methods of target-oriented syntheses to find molecules hitting preselected protein targets or diversity-oriented syntheses to find molecules modulating particular pathways without regard to a predefined protein target should enhance the ability of chemists to generate compounds relevant to new mechanisms, ${ }^{27}$ but the challenge of library diversity remains real. Additionally, rational methods of matching protein structure to molecular design are based on crystallized proteins that are likely to have a different shape from their configuration in vivo and hence are likely to be misleading. ${ }^{28}$

\section{Animal models}

Modern pharmaceutical discovery is heavily dependent on various disease models yet serious fundamental questions can be raised about the adequacy of these models in general. A great deal of work has gone into characterizing many different animal models for a variety of CNS diseases in terms of understanding their face validity, predictive validity, the phenomenology of ab- normalities observed in the model, and construct validity, among other qualities ${ }^{29}$; yet as Horrobin ${ }^{30}$ has noted, for any animal model to be relevant to the development of treatment for a disease, at least three logical conditions should be met, but they rarely are. First, one should understand the nature of the animal model in detail (i.e., understanding comprehensively why the pathology of the animal model occurs and the mechanisms and systems biology). Second, one should understand the nature of the human disease in detail (i.e., why the pathology of the human disease occurs and the mechanisms and systems biology). Third, one should know that the animal model and the target disease in humans are congruent in all important respects, because if the animal model and the target human disease are not congruent in some important aspect, it is very likely that predictions made with the animal model will be wrong. In some cases (e.g., neurotrauma or some stroke models), a plausible case can be made for reasonable congruity between model and human disease. Head trauma models can mimic the process that induces lesions in humans within the limitations of cross-species differences in reaction to trauma, and various methods of stroke induction, from ligation to introduction of clots or microspheres, can induce lesions similar to those experienced by human stroke patients. Even in such cases, however, there are still differences in circulation, species-related responses, the size of the organ affected, and the time course of lesion induction that may be relevant to the appropriateness of the model.

In most neurologic diseases and almost all psychiatric disorders, the gap between animal model and human disease is great and the animal models used in neurotherapeutics development do not come close to meeting the three conditions. Consider the following examples. First, studies of the biologic effects of molecules focus on effects on cells in cell culture, cells with a very different relation to nutritional systems and to their exogenous environment, and cells with a generally different phospholipid composition in their membranes than cells in vivo. ${ }^{28}$ Yet the phospholipid composition of a membrane can easily affect the shape and hence the binding characteristics of molecules embedded within them and the relations to nutrients and the environment are rather different than in in vivo cells with a bloodbrain barrier. Further, in vitro models typically remove the relevant region from potentially important regulatory controls. Second, genetic animal models of disease (for example, animals with gene deletions) are often posited as indicators, allowing a more comprehensive understanding of the relevance of a system to a given disorder. Yet when the details are examined, a given gene deletion may have markedly different effects in different strains of mice. ${ }^{31}$ If the deletion in a given mouse cannot universally predict the outcome in all other strains of mice, how likely is it to predict the outcome in a human $?^{30}$ This 
is not to dismiss the remarkable science and potential utility of transgenic mice, but rather to suggest that the biologic complexity is such that the congruence of animal and human models is difficult to establish. However useful, these models are maps with potentially important differences from the territory of the human disease. Third, regulation and metabolism may vary across species even if certain pathways are preserved. Fundamental problems with animal models occur across all therapeutic areas but the very complexity of the CNS and the size and behavioral uniqueness of the human brain make the problem of congruity loom large for neurotherapeutics developers. In this sense, animal models for most human CNS diseases at best can be interpreted as having suggestive value, indicating that a potential hypothesis should be tested in humans. The clear caveats are that in neurotherapeutics development the distance between model and man is large and that human data are far more likely to be of relevance and use than data from even multiple animal models.

A further source of incongruity arises from the fact that interventions in the animal models are often used in a manner very different from the human model. In terms of face validity of the model, stroke research could be considered a good area for extrapolating from animal data to humans. A stroke intervention may be tested in an animal using an agent administered before stroke induction, but is then tested in a human after a stroke occurs. A stroke intervention in animals may be evaluated by rapid inspection of the volume of brain infarcted, then tested in man by using a behavioral rating scale at a very different assessment time. Important and practical reasons are always cited for why the animal assessment has to be done in a different way than the human disease assessment but the reality is that these differences rarely benefit the predictive validity of the model. In the case where the animal model does potentially have clear significance to the human disorder, investment of effort in developing the model to allow testing similar to the planned timing in humans is appropriate.

Many of the most important neurotherapeutic challenges, such as depression, psychosis, and dementia, derive from disorders with relatively subtle pathology that are detectable only by detailed neuropsychiatric evaluation. Conversely, many of the animal models used to assess compounds for neuropsychiatric disorders have at best a passing plausibility in reflecting on a given disease and no solid mechanistic foundation to suggest congruity. Models that have shown an ability to detect an effect generated by a molecule known to be clinically effective in treating a disease may well be used as screens for subsequent potential therapeutic molecules even if the connection between the effect detected and the mechanisms causing the disease is unproven. The flaw is that the effector molecule may produce the effect in the model for a reason different from the mechanism that actually was responsible for its therapeutic effect in humans. The animal model may be a model for detecting the molecular perturbation caused by the class of compounds in which it is initially validated, but that utility says nothing about its ability to detect the utility of a class of molecules operating by a novel mechanism. Looking outside the nervous system to progress in diseases such as asthma, what initially was conceived as a single syndrome is slowly evolving into a complex cluster of diseases with a variety of underlying genetic susceptibilities, molecular mechanisms, and environmental triggers that are leading to a focus on distinct underlying disease states such as atopy and airway inflammation. ${ }^{32}$ One could easily foresee a similar pathway for a syndrome such as depression or schizophrenia, with subsequent fractionation of the population into distinct causal subcategories. In such cases, animal models that are genuinely mechanistically relevant to the disease of interest could then be developed.

\section{Trial conduct}

The challenges in the conduct of neurotherapeutic trials are similar to the challenges experienced in trials in other specialties. Clinical trials themselves are in general more similar as project management exercises, but neurotherapeutic trials do present some unusually intense challenges compared with other areas.

First, extra science is needed with neurotherapeutics to have confidence that the drug is being tested in adequate doses in the target organ. With most therapeutic trials the target organ is in free communication with the circulation and the free concentrations of the molecule generally reach the target organ. In neurotherapeutic trials, however, the blood-brain barrier acts as a distorting influence capable of transporting or blocking a particular therapeutic. If arterial concentrations are sustained for a sufficiently long period, the central compartment should come into equilibrium with the free unbound plasma concentration, but a variety of sink conditions maintained by $\mathrm{pH}$ gradients across cell membranes, metabolism, and active transport, among others, can prevent it. ${ }^{33}$ Neurotherapeutics developers must remember that while peripheral compartment pharmacokinetic and pharmacodynamic parameters are useful in understanding adverse events, the time course and extent of exposure of the central compartment are the main areas of interest for efficacy. Animal models of blood-brain barrier effectiveness can be useful in assessing the extent of this effect, but vary in their predictions and are typically not done in a manner that allows understanding of the relationship between the concentrations of free compound in the peripheral compartment and the simultaneous concentrations in the central compartment. 
In principle, it is possible to estimate the concentration and time course of CNS exposure to a molecule by making direct measurements of concentrations in the cerebrospinal fluid or by indirectly measuring binding of labeled molecules to brain or displacements of labeled molecules from brain. The "dynabridge" methodology, using continuous cerebrospinal fluid sample collection concurrently with sample collection from the peripheral compartment and correlating the results with behavioral effects, is one interesting approach to this problem that has been used in the assessment of a neuropsychiatric compound. ${ }^{34}$

An alternative approach to this problem is to develop PET ligands that allow the conduct of PET studies to determine brain binding. Within pharma, the combination of scientific tasks and contractual/financial arrangements needed to generate materials to conduct PET studies can easily consume 12 to 18 months. An organized effort to develop the appropriate ligand, beginning long before human testing, is required because these data are most useful early in human testing, to assist in dose selection and to have full confidence that an adequate concentration of the compound has been tested; the conduct of such studies is now common but not routine. The risk of conducting clinical studies in humans without performing the experimental work to understand what dose and timing is required to achieve a mechanistically appropriate concentration in the human central compartment is that a molecule that could actually work might fail in human testing because it never reached the needed concentration at the target organ or it reached the relevant concentration in a time frame different from the serum concentration and was not assessed at the right time. An opportunity cost also results from failing to attend to the development of appropriate neuroimaging technologies. PET technologies provide qualitative and quantitative $\mathrm{e}^{35}$ physiologic information and allow a wide variety of biological markers to be quantified in the human brain. ${ }^{36,37}$

Second, the assessment of treatment effect can be particularly complicated in neurotherapeutic trials. The brain is complex. An intervention may have different effects on multiple particular brain regions, reflected in differential impact on $N$ different dimensions of brain function. For example, a dopamine agonist may produce psychiatric effects by its mesolimbic activity, but may also increase prolactin secretion and produce galactorrhea by its tuberoinfundibular blocking activity and motor effects by its activity on the basal ganglia. More subtly, a D2 superfamily dopamine antagonist may differentially bind D2, D3, and D4 receptors centered in different brain regions, producing complex interacting cognitive and affective effects. An assessment instrument such as a rating scale may have more items that measure one aspect of brain functioning than another or may weight them differently, and different rating instruments for the same disease may also give more or less weight to one aspect of brain function than another. Hence each treatment has $N$-dimensional effects and each assay instrument potentially samples some of those dimensions better than others. Consequently the assay instrument that best detects the effects of one compound may not be the best one to detect the effects of another compound affecting the disease in a different way. Thus early depression rating scales developed around optimizing assessment of tricyclic antidepressants may not be optimal for assessing the effects of a novel treatment, even if that treatment is more effective on critical aspects of the disease.

In understanding a molecule, this phenomenon points out the importance of using a range of assessment instruments during early development to better understand the parameters of function influenced by the therapeutic agent. A skilled focus on exploratory data analysis evaluating where the various instruments agreed and where they detected differences may help understand how the compound affects the disease and what assessment instruments best detect the compound's effects. As a practical matter, for a novel therapeutic it is desirable to have an efficiently executed trial with near real-time availability of data from sophisticated assessments to facilitate ongoing exploratory analysis of the effects of the compound. A trial design for non-pivotal trials that is doubleblind (patient and investigator blinded) but not tripleblind (company and academic advisors able to conduct unblinded exploratory analysis) might optimally allow prolonged thought, analysis, and discussion to assist downstream design of subsequent trials without adversely affecting the critical path timelines that are essential to submission and eventual retention of patent life.

Third, determination of dose, formulation, and regimen is particularly challenging in neurotherapeutic trials. In anti-infective trials, the general level needed to suppress or kill a particular organism in vivo may closely mimic the relevant concentrations from in vitro studies. In neurotherapeutic trials, however, in addition to the aforementioned concerns with penetration to the target organ, assessment of effect is also problematic. Assessments are done at a particular time and often are made with instruments that are statistically highly variable because of circadian factors, the interaction between the investigator and patient, and the fact that compound effects on the brain may have a different time course than serum concentrations.

Finding the optimal dose for a compound is very difficult. Recent developments in statistical design, combined with the availability of central randomization controls create the possibility of improving this problem by using adaptive designs in trials. Normally in dose-rang- 
ing trials some number of doses and a placebo are allocated across a target number of patients either in equal proportion or in some fixed ratio. Modern technological developments combined with near real-time databases allow for an alternative approach. A trial could be conducted with some fraction of the total number of patients being allocated to each arm. Those patients can then be rapidly assessed for effectiveness and/or safety. If the target is effectiveness, for example, after the initial cohort is treated, some relative efficacies may be noted in the various arms. These effects may be real or artifacts seen in small samples because of significant random variability. Nonetheless, the randomization schema could then be modified to have a higher probability of assigning patients to arms that are showing an effect and a decreased but nonzero probability of assigning patients to arms that show futility. As the trial proceeds, arms that initially look positive would be subjected to more stringent testing by preferentially having more patients assigned to them. If the compound is genuinely efficacious, these arms will likely hold up and show a more robust and well-tested effect. If these arms appeared more positive purely by chance, the additional number of test subjects would likely dilute the chance effect and show it as spurious. As ineffective arms reached the point of futility, i.e., having no significant chance of showing an effect that would reach significance, they would be closed or have fewer patients assigned to them, allowing the other patients to be assigned to arms that have a better chance of detecting an effect. In some cases such an approach could be completely automated and blinded and likely fully acceptable for pivotal registration trials. More complex modifications may require guidance by a monitor and could potentially be useful, but unblinding and human intervention could also lead to regulatory rejection as biased. The regulatory and statistical risks of automated or manually assisted adaptive randomization clearly require careful study-by-study consideration, but the benefits-in time, money, and reduction of risk to human subjects—are potentially great.

\section{The Biodesign strategy}

A mix of innovative approaches being tried in drug development as separate pieces have the potential to act synergistically to resolve or ameliorate some of the economic and scientific challenges discussed above. Considered together, a new strategy, Biodesign, has potential to allow us to progress past the limitations of the current rational design strategy centered entirely on small molecules. The term Biodesign does not equally apply to all components of this new strategy but it seems apt nonetheless in that the focus is on using probes generated biologically to assess human disease. Four components of this strategy will be discussed separately, then the synergies of their combination will be considered along with an assessment of the major impact this model may have on the future of the therapeutics development partnership between academicians, industry, and regulators.

\section{DISEASE MODELS}

In biologic reality, every pathological process is the physical result of a dysfunction or of multiple interactive dysfunctions of one or more processes. Diseases are thought initially to be the result of one dysfunctional system, which is explored and found to have some degree of relevance. As the science evolves, other systems affecting the disease are discovered. Interactions between the systems are explored and the literature becomes dense with the myriad details and hypotheses underlying the integrated pathophysiological process of the disease. Scientists focused on a particular disease have their own mental maps of the key processes and interaction points based on their own scholarly reviews, the reviews of others, and the particular window on the disease opened by their own research experience. Predicting which interventions will actually work to change the manifestations of a disease is a difficult exercise. Systems show biological redundancy and regulatory control mechanisms can be multiple and complex. Blocking one path may not produce the desired result because alternative paths compensate. In no therapeutic area is this more problematic than in CNS disorders. The time scales of drug effects may be very different from the time scales relevant to the pathology underlying the disease. Integrating these and other relevant variables to predict response is a major challenge.

Disease models essentially attempt to scour the literature for all relevant information bearing on known aspects of the pathophysiology of a disease and explicitly synthesize the lines of causal events to create a model that dynamically describes all relevant regulatory mechanisms of the disease process and links elements by differential equations. ${ }^{38}$ By integrating clinical, genomic, proteomic, physiological, and other biological data in silico in a computerized platform, it should be possible to capture the control principles of the system with everhigher degrees of completeness. ${ }^{39}$ The model can be tested by assessing predictions against experimental data and can be enriched by the ongoing addition of experimental data. In a sense, the models derive from "reverse engineering" of the disease, identifying known manifestations, reasoning back to the relevant causal mechanistic pathways, nesting detailed subsystems with control and context defined by the behavior of the larger disease, incorporating new relevant pathways as they are discovered, and explicitly mapping how perturbations of underlying causal pathways should impact on the disease. ${ }^{38}$ With the initial set of conditions representing the initial disease state as a baseline, the goal is to simulate the 
subsequent behavior of the system in a process termed biosimulation ${ }^{40}$ to allow researchers to ask "what if" questions of the model.

An analogy has been made to the complex process of airplane design: "If Boeing made airplanes the way the pharmaceutical industry makes drugs, you'd see them making many different designs, flying them, and then mass-producing those models that didn't crash." ${ }^{41}$ Such a model would not work economically for Boeing and it is not working optimally for pharma. Better use of the ability to assist the effectiveness of human intellect by empowering and embodying it with explicit models is one underpinning of the future of pharma. In the Biodesign paradigm, the disease model is the intellectual core used to integrate all data collected from in vitro, animal, and human experimentation. The predictions obtained from disease modeling are only as good as the model but the explicit nature of the model facilitates correction when erroneous predictions are made. Doubtless development of an effective disease model for any disorder, from epilepsy or Parkinson's disease to panic disorder or attention deficit disorder, would be a financially and intellectually intense and expensive investment, but the power of the model to suggest increasingly more powerful interventions would well cover its costs.

One important implication of the Biodesign strategy is that companies would focus on disease areas rather than on molecular platforms and would invest heavily in developing increasingly sophisticated disease models in their areas of interest. In fact, a highly sophisticated and developed disease model would become the core intellectual property of the pharmaceutical company (much as the patent estate is now) and the center of deliberations internally and with academic collaborators. The possession of a sophisticated model would allow synergistic interaction between discovery and clinical colleagues because it would constitute an integrative mechanism, combining the results of animal data collected with the result of assessment of various probes. Moreover, such a model would suggest interventions to be tried in humans and could be modified as the results of those interventions are captured from patients with different stages and severities of the target illness.

Such models would have multiple applications. One of the most promising applications of this approach is to examine the logic of a disease and to predict which interventions at which part of the disease would be most likely to produce desired downstream effects on disease manifestations, facilitating the otherwise lengthy target validation and selection aspect of pharmaceutical development. Another intriguing aspect of this approach is that it potentially offers an opportunity to assess the impact of agents that affect multiple pathways to see if they are synergistic, thereby presenting a possible solution to the specificity challenge noted previously.
An underlying advantage of the disease model is that it forces a movement away from simplistic and reductionistic models of function and disease and toward an integrative model. Disease is a reflection of complex processes and the more explicit the processes can be made, the better the chance for a rational intervention. One might picture the truth as an intricate stained glass window that has been shattered. A person focused on a particular mechanism or process can look through a fragment and claim, "I see the truth and the truth is green," but another can disagree, saying "I see the truth and the truth is red." Both are right and neither is right. The truth is a complex composite that contains the information of both but exceeds it by the interaction of the pieces. Although singular geniuses may be able to attain a scholarly comprehension exceeding a model, the model has the advantage of allowing collaborative progressive hypothesis generation, testing, and growth, and does not preclude enrichment with the insight from singular geniuses.

\section{EXPERIMENTAL MEDICINE}

Experimental medicine groups within pharma perform a variety of roles. In the context of the Biodesign strategy, the role of experimental medicine is to develop the methodologies and tools needed to safely answer key questions about disease processes in humans, to strengthen the disease model, and to undertake studies in humans intended to assess the biological impact of therapeutic interventions.

On one level, many experimental medicine questions fall into the realm typically associated with clinical pharmacology departments. Is the compound absorbed and what is its concentration time course? Does the compound penetrate to the desired site of action for the appropriate period of time and at the desired concentration? For example, does an antagonist penetrate to the CNS compartment at concentrations sufficient to produce the blockade desired? Does the compound mechanistically do what it is intended to do? For example, if the compound is a cyclooxygenase (COX)-2 inhibitor, does it in fact show evidence of inhibiting COX-2 in the target organ? Does the compound exert a biological effect? In the best of all possible worlds, one would want to know if it produces a biological effect that is specifically causally relevant to the disease of interest. Because the full mechanistic causal chain is not always known in CNS diseases, it may not be possible to specify a key causal element. If the compound does not produce any biological effect on systems it should affect and does not mimic biological effects that were important causally in the animal models used for earlier assessment; however, the probability of producing a profound effect on disease seems less than optimal.

On another level, however, the role of experimental 
medicine goes beyond that of a typical clinical pharmacology assessment by assessing risk factors in an intervention. Many therapies are known to have class-associated limiting toxicities. For example, some thiazolidinediones are known to be associated with varying degrees of hepatotoxicity. ${ }^{42}$ If a compound of this class is being developed, it would make sense to perform the key experiments needed to rule in or rule out the possibility that a compound has an unacceptable level of class-associated limiting toxicities very early in development. This would better protect larger numbers of future subjects from exposure to toxicity and reduce the probability of expensive late-stage failure.

In the Biodesign model, experimental medicine is actively involved in further developing the disease model. It would collaborate closely with discovery, analytical chemistry, a biomarker laboratory, and clinical pharmacology functions in developing sophisticated technologies to allow collection of information about the compound and its effects on human physiology and disease relevant to the model. Information from pharmacokinetic and pharmacodynamic relationships in the peripheral and central compartments could be assessed using markers derived from biochemical, neurophysiological, pharmacodynamic, or neuroendocrine biomarkers, or from imaging technologies such as PET, single-photon emission tomography, and magnetic resonance spectroscopy, or on functional brain imaging or assessment technologies such as magnetic resonance imaging, electroencephalography, or magnetoencephalography. Clinical staff familiar with the human disease, academic advisors familiar with the disease and with the biology underlying the selection of biomarkers, and discovery scientists familiar with the results of animal models all have significant input into the model and into the design of experiments, but it is also true that the detailed design and conduct of these highly specialized trials requires intense expertise in itself, expertise that needs the organizational support and rootedness that an experimental medicine group provides.

\section{BIOLOGICAL TECHNOLOGIES}

The third part of the Biodesign strategy centers on the development of protein probes to test normal physiology and disease in humans both to enrich the model and the insight it yields into the targets for therapy and to potentially become therapies in themselves. The potential for biologics both as drugs and potential probes has been recognized. ${ }^{32}$ The major weight of pharma personnel and financial resources have focused primarily on the small molecule side, on producing "white powders" of highly defined molecules rationally designed and created to hit some specific target or targets. The rise of the biotechnology industry, with its intense focus on protein thera- peutics over the past 20 years, has been an important complementary development. In 1999, a total of 59 recombinant proteins and monoclonal antibodies were available. ${ }^{43}$ In 2001 , more than $35 \%$ of the 37 new active substances launched were protein therapeutics. ${ }^{44}$ The power of rationally engineered proteins is becoming increasingly evident.

The development of protein therapeutics can begin with a naturally occurring protein acting as a preselected "lead" compound. However, a natural protein evolved by nature for one role and the ideal protein therapeutic may have some additional characteristics. For this reason, the lead protein may be modified to produce variant proteins as a therapeutic with more desirable characteristics that influence key features such as mechanism of action, efficacy, adverse events, stability through purification, formulation, storage and administration, solubility, and production costs. Routes of modification may include sitedirected mutagenesis and phage display. For example, structures can be modified to become substantially more robust to oxidative stress, changes in solution and $\mathrm{pH}$, and temperature, or to replace cysteines with serines to reduce disulfide bond formation. Similarly attachment to polyethylene glycol, fusion to proteins with long serum half-lives, alteration of oligomerization state, glycosylation, and modulation of receptor-mediated uptake and turnover can markedly alter the pharmacokinetic properties of a molecule. ${ }^{45}$ Many engineered molecules are currently being marketed, e.g., aldesleukin (Proleukin), interferon $\beta-1 \mathrm{~b}$ (Betaseron), insulin lispro (Humalog), insulin aspart (NovoLog), and peginterferon alfa- $2 b$ (PEG-Intron).

As technologies have developed, the opportunity to manipulate the protein sequence and/or composition, driven by specific hypotheses, has increased. ${ }^{45}$ It is remarkable that rational protein design has progressed to the point where proteins can be produced with novel mechanisms of action. For example, interleukin- 6 and vascular endothelial growth factor, both 4-helix bundle cytokines, have been engineered to function as receptor antagonists rather than agonists. ${ }^{45}$ One concern with protein therapeutics, particularly therapeutic proteins derived from non-human sources, has been the development of harmful immune responses. Increasing interest has focused on deriving therapeutics from human proteins that are less likely to stimulate an immune response. Approaches to date include humanization of murine antibodies, mutagenesis of peptides that bind class II major histocompatability complex alleles, and mutagenesis of epitopes in the protein structure that are particularly important in stimulating the immune response.

The rational design of protein therapeutics has the potential to use systematic and quantitative engineering approaches $^{46}$ for structure-based sampling followed by 
high throughput screening of a protein library. As hypotheses are identified, probes must be generated. In the long run, small molecules may be ideal for purposes of manufacture and marketing. However, the speed of development of protein therapeutics argues for a strong capacity to rapidly generate the molecules as probes and potentially as therapeutics. For example, if an antagonist for a particular molecule is required, monoclonal antibody probes for testing the hypothesis might be more rapidly generated than small molecules could be synthesized and allow hypothesis testing with a greater assurance of safety. As interest focuses on new molecular targets that are very different from the serine proteases, G-protein-coupled receptors, and similar families of targets on which most pharma libraries were developed, the structural diversity of protein therapeutics might be used to create antagonist and agonist compounds as powerful probes. The structural diversity of the protein probes and the increasing ability to modify and design their functionalities will facilitate a biological way to design therapeutics. These probes may develop into therapies in their own right but also serve the purpose of validating whether a particular target is worth pursuing with the resource-intensive processes needed to develop small molecule therapeutics. Because failure rates for new targets are high, by eliminating the poor targets more quickly the protein probes would facilitate a more focused and cost-effective small molecule effort.

Protein therapeutics have additional attractions as probes and as therapies. Protein therapeutics can be developed more quickly than small molecules, in the range of 2 years rather than 4 years. While pharma discovery libraries may have a limited number of potential shapeprobes to test against new genomic targets, protein therapeutics are capable of rapidly generating extraordinary shape diversity. The Cambridge Antibody Technology Group has created a library of approximately 10 billion different molecules, ${ }^{32}$ a size that dwarfs any other small molecule library. And immunogenicity aside, protein therapeutics are degraded by normal proteolytic processes, reducing the problem of active metabolites, which complicates the development of small molecule therapeutics. The manufacture and cost of goods of protein therapeutics presents challenges and corresponding economic advantages in terms of not being as susceptible to generic competition, which complicates their economics as therapeutics, but their speed of and safety for development as probes is clear. The penetration of protein therapeutics across the blood-brain barrier is a significant challenge but one that can be addressed; protein therapeutics have been shown to penetrate to the brain and in an animal stroke model prevented the slow progressive death of neurons. ${ }^{47}$

\section{MEETING THE CHALLENGE WITH BIODESIGN: IMPLICATIONS FOR THE FUTURE}

The use of protein-based probes and therapeutics as a basis for the evolution of the pharmaceutical industry has been considered. ${ }^{32}$ A target molecule can fail in more ways that one. More than a thousand different problems have been noted in the gene underlying cystic fibrosis. As highly targeted protein therapies are developed, the initial molecules will most likely target the most common defects. Subsequent lifecycle management will likely consist of developing additional molecules that target successively less-common molecular defects. These new therapies might be independent products or might be added to a cocktail, as study results indicate. Therapies are likely to be highly individualized and may be delivered through specialists rather than generalists. The corporate structures based on marketing high-volume blockbuster compounds that work overall for a population but fail in many subjects will be replaced by structured delivery of high-utility therapies with markedly superior efficacy to smaller numbers of patients for a given therapy. The structure of marketing, advertising, and relations with healthcare providers and patients will change markedly in an environment of individualized medicine. Advertising may play a lesser role and sophisticated diagnostics a greater role in determining which treatment a patient receives.

An underlying theme of all of these considerations is the need for greater intelligence in the way neurotherapeutics are developed. We need to better understand the target diseases, animal models, the relation between the models and the diseases, and the interaction between therapeutics and the diseases and models, and generally have a more thoughtful, explicit, hypothesis-driven approach to therapeutics development. While the intellectual assets within pharma are significant, intellectual assets in academia and in the clinic are just as important. With the shift in focus from platform-based intellectual property to disease-model-based intellectual property, pharma needs to engage the intellect of a wide base of disease experts outside the corporate world. Conflicts between academic responsibilities and corporate confidentiality can and must be addressed in a manner fair to the involved entities and society. The cost of failure is too high to permit anything less than engaging the best minds. Development of better remote-presence interactions such as internet-assisted meetings, methods for secure sharing of electronic data, and alternative employment relationships such as retainer-based hiring of academicians or investigators seem likely. Companies unable to move beyond the "not invented here" mentality will succumb in the competition with companies that are open to engaging the best and the brightest. 
Another key implication for this model is that the relationships between regulators and developers will develop more as an interactive partnership. Why? The nature of the Biodesign model includes a lot of early hypothesis testing and developmental work conducted in healthy subjects and volunteer patients as the model is developed and its predictions are tested. Investigators and developers are responsible for conducting well-designed, safe, and ethical research, but regulators have a key oversight responsibility for ensuring public safety and accountability in the process. An ongoing interactive, consultative relationship in which the data are fully and transparently available to regulators is the best way to ensure public safety. Data management will likely rely on a rolling, continuously updated database with transparency of analysis and built-in electronic fidelity measurements such as audit trails and access controls. The continuous safety evaluation of therapeutics based on some sort of sampling access to electronic medical records with appropriate privacy safeguards is also a likely point of collaborative interaction and dialectic between therapy developers and regulators.

\section{SUMMARY}

Stakeholders in neurotherapeutics development-patients, advocates, clinicians, academicians, industrial developers, and regulators-all want therapies that are more effective, safe, and cost-efficient. The current explosion of scientific knowledge presents an incredible opportunity to create the needed new generation of therapies. The current system of pharmaceutical development is plagued by low rates of success, huge costs, long cycle times, and more fundamental challenges in determining how to incorporate a future of individualized therapies into a workable business model. Economic challenges are matched against equally severe scientific challenges with molecular diversity, compound specificity, animal models, and trial conduct. A series of integrated strategic changes, the Biodesign strategy, presents a potential solution to this challenge by building future corporate discovery strategies around a core informational approach focused on a sophisticated disease model coupled with hypothesis testing. Experimental medicine's capacity to use biologically designed protein therapeutic probes has the potential to build future pharma on highly diverse compounds with any range of specificities nature can generate for testing in animals and humans linked to the model. Molecules so developed and vetted will enter into full-scale clinical testing without many of the problems that underlie current low success rates and with higher safety parameters. Effective trial execution using the best of modern adaptive design approaches should allow confidence in results assessment if the mechanism being tested lives up to its mechanistic promise with clinical results in the selected relevant population. Subsequent development may proceed with the protein therapeutic and/or may be accompanied by a small molecule development program in parallel.

\section{REFERENCES}

1. Drews J. Drug discovery: a historical perspective [comment]. Science 287:1960-1964, March 172000.

2. Hensley S. Proteins-not genes-could be clue to human complexity, disease. Wall St J 2001.

3. Pharmaceutical Research and Manufacturers of America (PhRMA). New medicines in development for mental illness, Washington, DC: PhRMA, 2002.

4. Pharmaceutical Research and Manufacturers of America (PhRMA). The value of medicines 2001. Washington, DC (http:// www.phrma.org/publications/)

5. Ashton GA, Joshua PJ. Industry Success Rates 2002-including trends in success rates, ref number CMR02-175R. Surrey, United Kingdom: Centre for Medicines Research International Limited.

6. Grabowski H, Vernon J, DiMasi J. Returns on research and development for 1990 s new drug introductions. Pharmacoeconomics 20[Suppl 3]:11-29, 2002.

7. Tufts Center for the Study of Drug Development (Tufts CSDD). R\&D efficiency. Outlook 2003. p 2, 2003.

8. DiMasi JA, Hansen RW, Grabowski HG. The price of innovation: new estimates of drug development costs. J Health Econ 22:151$185,2003$.

9. Tufts Center for the Study of Drug Development (Tufts CSDD). Impact report. 5(3):1, May/June, 2003.

10. Peck CC. Drug development: improving the process. Food Drug Law J 52:163-167, 1997.

11. Ogg MS, van den Haak MA, Halliday RG. Activities of the international pharmaceutical industry in 2000: pharmaceutical investment and output. CMR International (http://www.cmr.org/index.htm).

12. Grabowski H, Vernon J. Returns to R\&D on new drug introductions in the 1980s. J Health Econ 13:383-406, 1994.

13. A plan to ensure taxpayer interests are protected. NIH response to the conference report request for a plan to ensure taxpayers' interests are protected. Department of Health and Human Services, National Institutes of Health (http://www.nih.gov/news/070101wyden. htm), 2001.

14. The Pharmaceutical Research and Manufacturers of America (PhRMA). Annual Survey, 2001. Pharmaceutical Industry Primer 2001, p 5. 2001.

15. The Pharmaceutical Research and Manufacturers of America (PhRMA). Annual Survey, 2001. U.S. FDA. Global Market Res \& Analysis, 2001.

16. IMS Health, 2001 in Pharmaceutical Industry Primer 2001, PhRMA p 8, 2001.

17. Peakman, Franks TS, White C, Beggs M. Delivering the power of discovery in large pharmaceutical organizations. Drug Discovery Today 8:203-221, 2003

18. Beggs M. HTS -where next? Drug Discov World 2:25-30, 2000.

19. Beggs M, Long AC. High throughput genomics and drug discovery-parallel universes or a continuum? Drug Discov World 3:7580, 2002.

20. Lin L. Betaseron. Dev Biol Stand 96:97-104, 1998.

21. U. S. International Trade Commission, Global Competitiveness of U. S. Advanced-Technology Manufacturing Industries: Pharmaceuticals, Report to the Senate Finance Committee. Washington, D.C.: ITC September 1991.

22. Industry perspectives: U. S. outpaces Europe in growth of emerging biotech market. IMS Health (http://www.imshealth.com/ims/portal/ front/articleC/0,2777,6599_40183890_40053441,00.html), 2002.

23. Drews J. In: Human disease: from genetic causes to biochemical effects (Drews J, Ryser S, eds), pp 5-9. Berlin: Blackwell, 1997.

24. Drews J, Ryser S. Classic drug targets. Nat Biotechnol 157:1, 1997.

25. Doull, Iolo JM. Recent advances in cystic fibrosis. Arch Dis Child 85:62-66, 2001. 
26. Fucharoen S, Winichagoon P. Thalassemia and abnormal hemoglobin. Int J Hematol 76[Suppl 2]:83-89, 2002.

27. Schreiber SL. Target-oriented and diversity-oriented organic synthesis in drug discovery. Science 287:1964-1969, 2000.

28. Horrobin DF. Realism in drug discovery: could Cassandra be right? Nat Biotechnol 19:1099-1100, 2001.

29. Willner P. Methods for assessing the validity of animal models of human psychopathology. In: Animal models in psychiatry, I (Neuromethods, No 18) (Boulton AA, Baker GB, Martin-Iverson MT, eds), pp 1-23. Clifton, NJ: Humana Press, 1991.

30. Horrobin DF. Modern biomedical research: an internally self-consistent universe with little contact with medical reality? Nat Rev Drug Discov 2:151-154, 2003.

31. Pearson H. Surviving a knock-out blow. Nature 415:8-9, 2002.

32. Arlington S, Barnett S, Hughes S, Palo J. Pharma 2010: the threshold of innovation: future series executive summary. Somers, NY: IBM Corporation, 2002.

33. Rowland M, Tozer TN. Clinical pharmacokinetics: concepts and applications, Ed 3, p 142. Baltimore: Williams \& Wilkins, 1995.

34. Cutler NR, Sramek JJ, Kurtz NM, Murphy MF, Carta A. Accelerating CNS drug development, Chap 5, pp 143-149. New York: Wiley, 1998.

35. Feng D, Weidong C, Fulton R. FIPS: a functional image processing system for PET dynamic studies. In: Physiological imaging of the brain with PET (Gjedde A, Hansen, Soren B, Knudsen GM, Paulson OB, eds), pp 35-38. San Diego: Academic, 2001.

36. Carson RE, Channing MA, Vuong B-K, Watabe H, Herscovich P, Eckelman WC. Amphetamine-induced dopamine release: duration of action assessed with [11 C]Raclopride in anesthetized monkeys. In: Physiological imaging of the brain with PET (Gjedde A, Hansen, Soren B, Knudsen GM, Paulson OB, eds), pp 205-209. San Diego: Academic, 2001.

37. Smith DF, Gee A, Hansen SB, Moldt P, Nielsen EO, Scheel-
Kruger $\mathrm{H}$, et al. Serotonin release and reuptake studied by PET neuroimaging using fenfluramine, water, oxygen, fluorodeoxyglucose, NS2381, and its enantiomers in living porcine brain. In: Physiological imaging of the brain with PET (Gjedde A, Hansen Soren B, Knudsen GM, Paulson OB, eds), pp 237-247. San Diego: Academic, 2001.

38. Stix G. Reverse-engineering clinical biology. A peacetime dividend yields drug trials on virtual patients. Sci Am 288:28, 2003.

39. McKenna C, Sudbeck B. Assessing the impact of predictive biostimulation on pharma R\&D, Entelos, Inc. In: PAREXEL's pharmaceutical R\&D statistical sourcebook 2003/2004 (Mathieu MD, ed), pp 128-129. Waltham: PAREXEL International, 2003.

40. Michelson S, Scherrer D. Assessing the impact of predictive biosimulation on lead optimization current drug discovery (http://www. currentdrugdiscovery.com), p 23-26. http://www.entelos.com/news/ publications.html.

41. Dutton G. Modeling software for speeding discovery and reducing attrition. Genet Eng News 22, 2002.

42. Tolman KG, Chandramouli J. Hepatotoxicity of the thiazolidinediones. Clin Liver Dis 9:369-379, 2003.

43. Zipkin I, Michael A. BioCentury 7:1, 1999.

44. IMS Health. Growth in new active substances: how biotechnology drugs are making inroads (http://www.imshealth.com/public/ structure/dispcontent/1,27779,1203-1203-144043,00).

45. Marshall SA, Lazar GA, Chirino AJ, Desjarlais JR. Rational design and engineering of therapeutic proteins. Drug Discov Today 8:212-221, 2003.

46. Marshall SA, Lazar GA, Chirino AJ, Desjarlais JR. Rational design and engineering of therapeutic proteins. Drug Discov Today 8:212-221, 2003.

47. Asoh S, Ohsawa I, Mori T, Katsura K-I, Hiraide T, Katayama Y et al. Protection against ischemic brain injury by protein therapeutics. Proc Natl Acad Sci USA 99:17107-17112, 2003. 